\title{
Hyperon Beta-Decay and Axial Charges of the Lambda in view of Strongly Distorted Baryon Wave-Functions
}

\author{
H. Weigel* ${ }^{\dagger}$ \\ Center for Theoretical Physics, Laboratory for Nuclear Science and Department of Physics \\ Massachusetts Institute of Technology, Cambridge, Massachusetts 02139 \\ and \\ Institute for Theoretical Physics, Tübingen University \\ Auf der Morgenstelle 14, D-72074 Tübingen, Germany
}

\begin{abstract}
Within the collective coordinate approach to chiral soliton models we suggest that breaking of $S U(3)$ flavor symmetry mainly resides in the baryon wave-functions while the charge operators maintain a symmetric structure. Sizable symmetry breaking in the wave-functions is required to reproduce the observed spacing in the spectrum of the $\frac{1}{2}^{+}$baryons. The matrix elements of the flavor symmetric charge operators nevertheless yield $g_{A} / g_{V}$ ratios for hyperon beta-decay which agree with the empirical data approximately as well as the successful $F \& D$ parameterization of the Cabibbo scheme. Demanding the strangeness component in the nucleon to vanish in the two flavor limit of the model, determines the structure of the singlet axial charge operator and yields the various quark flavor components of the axial charge of the $\Lambda$ hyperon. The suggested picture gains support from calculations in a realistic model using pion and vector meson degrees of freedom to build up the soliton.
\end{abstract}

PACS: 12.39.Dc, 12.39.Fe, 13.30.Ce, 14.20.Jn

\section{INTRODUCTION}

The study of the axial current matrix elements (or quark spin structure) of the $\Lambda$ hyperon, which is an interesting subject in its own, has gained further attraction as it has been suggested [1] that polarized $\Lambda$ 's could be utilized to gain information about the proton spin structure, i.e. the nucleon axial vector matrix elements. For this to be a sensible program it is necessary that large polarizations of the up and down quarks, $\Delta U_{\Lambda}$ and $\Delta D_{\Lambda}$, in the

\footnotetext{
*Heisenberg Fellow

${ }^{\dagger}$ e-mail:herbert.weigel@uni-tuebingen.de
} 
iso-singlet $\Lambda$ carry over to the corresponding fragmentation functions. Although this is an assumption one would expect the non-strange $\Lambda$ fragmentation functions to be small if model calculations undoubtly showed that $\Delta U_{\Lambda}$ and $\Delta D_{\Lambda}$ were small. On the other hand, if model calculations indicated that $\Delta U_{\Lambda}$ and $\Delta D_{\Lambda}$ were large, it would provide sufficient motivation to study and measure these fragmentation functions [2,3]. Using results on the axial current matrix elements from deep-inelastic scattering as well as hyperon beta-decay data together with flavor covariance indeed results in sizable polarizations for the non-strange quarks, $\Delta U_{\Lambda}=\Delta D_{\Lambda} \approx-0.20$ together with $\Delta S_{\Lambda} \approx 0.60$ for the strange quark [1,2, [4]. The use of flavor covariance is motivated by the feature that the Cabibbo scheme [5] utilizing the $F \& D$ parameterization for the flavor changing axial charges works unexpectedly well 6] as the comparison in table [ exemplifies. In the present note we will study an approach which allows the incorporation of deviations from the flavor symmetric formulation, after all $S U(3)$-flavor is not an exact symmetry. Clearly, any model that reproduces the data equally well as the Cabibbo scheme with a minimal set of parameters can be regarded as a reasonable description of hyperon beta-decay.

TABLE I. The empirical values for the $g_{A} / g_{V}$ ratios of hyperon beta-decays [7], see also [6]. For the process $\Sigma \rightarrow \Lambda$ only $g_{A}$ is given. Also the flavor symmetric predictions are presented using the values for $F \& D$ which are mentioned in section III. Analytic expressions which relate these parameters to the $g_{A} / g_{V}$ ratios may e.g. be found in table I of [B].

\begin{tabular}{c||c|c|c|c|c|c}
\hline \hline & $n \rightarrow p$ & $\Lambda \rightarrow p$ & $\Sigma \rightarrow n$ & $\Xi \rightarrow \Lambda$ & $\Xi \rightarrow \Sigma$ & $\Sigma \rightarrow \Lambda$ \\
\hline emp. & 1.258 & $0.718 \pm 0.015$ & $0.340 \pm 0.017$ & $0.25 \pm 0.05$ & $1.287 \pm 0.158$ & $0.61 \pm 0.02$ \\
$F \& D$ & 1.258 & $0.725 \pm 0.009$ & $0.339 \pm 0.026$ & $0.19 \pm 0.02$ & $1.258=g_{A}$ & $0.65 \pm 0.01$ \\
\hline \hline
\end{tabular}

Our treatment of flavor symmetry breaking is based on the Skyrme model approach to describe baryons as solitons in an effective meson theory. In this type of models baryon states are obtained by quantizing the large amplitude fluctuations (zero modes) of the soliton. In the proceeding section we will briefly review the quantization procedure with the inclusion of flavor symmetry breaking. This approach is of great fundamental interest not only for the large $N_{C}$ treatment of QCD but certainly also in its own right; especially because this approach has been (at least quantitatively) successful in understanding the proton spin problem [9 11]. Furthermore, in the framework of quantizing the soliton the study of flavor symmetry breaking in these models is very interesting. This is even more the case as some of the difficulties encountered earlier (such as the overall scale in the predicted baryon mass differences [12, 13] or the unexpectedly large strangeness contribution to nucleon matrix elements [14,9]) have been largely understood and solved [15, 11, 16]. It is thus appealing to also study the $\Lambda$ axial charges in such a framework, especially because they might be accessible experimentally [4, 17, 18]. Here we will focus on a description with the symmetry breaking mainly residing in the baryon wave-functions, including important higher order contributions. The order parameter is the strange current quark mass, $m_{s}$. In the effective meson Lagrangian it emerges via the meson properties, e.g. $m_{K}^{2}-m_{\pi}^{2}=\mathcal{O}\left(m_{s}\right)$. Sizable deviations from flavor symmetric (octet) wave-functions are needed in the chiral soliton approach to account for the pattern of the baryon mass-splittings [11. The proposed picture implies that the strange quark component in the sea is suppressed, a scenario which 
has also been considered in ref [19]. On the other hand we will assume that the current operators, from which the charges are computed, are dominated by their flavor symmetric components. We will find that the proposed approach approximately reproduces the data with no (or minimal) explicit symmetry breaking in the axial charge operator. The present studies represent a refinement of some earlier calculations as we now include contributions to the axial charge operator which were omitted in ref [ [ ] and are subleading in the $1 / N_{C}$ counting. A systematic expansion in $1 / N_{C}$ would also require a careful treatment of the allowed representations in flavor space for the baryon wave-functions. We do not attempt such an expansion but rather assume the physical value $N_{C}=3$. In addition we present the results obtained from a complete calculation in a realistic vector meson soliton model. That calculation supports the suggested picture.

\section{SYMMETRY BREAKING IN THE BARYON WAVE-FUNCTIONS}

Here we briefly review the energy eigenvalue problem for the low-lying $\frac{1}{2}^{+}$and $\frac{3}{2}^{+}$baryons as it arises in the collective coordinate treatment of chiral soliton models. This approach was initiated in ref [15]. In the model framework it leads to exact eigenstates for an arbitrary strength of the flavor symmetry breaking. The collective coordinates for flavor rotations are introduced via

$$
U(\vec{r}, t)=A(t) U_{0}(\vec{r}) A^{\dagger}(t), \quad A(t) \in S U(3) .
$$

$U_{0}(\vec{r})$ describes the soliton field configuration embedded in the isospin subgroup of flavor $S U(3)$. A prototype model Largangian for the chiral field $U(\vec{r}, t)$ would consist of the Skyrme model supplemented by the Wess-Zumino-Witten terms as well as suitable symmetry breaking pieces. In the action notation it reads

$$
\Gamma=\int d^{4} x\left\{\frac{f_{\pi}^{2}}{4} \operatorname{Tr}\left[\partial_{\mu} U\left(\partial^{\mu} U\right)^{\dagger}\right]+\frac{1}{32 \epsilon^{2}} \operatorname{Tr}\left[\left[U^{\dagger} \partial_{\mu} U, U^{\dagger} \partial_{\nu} U\right]^{2}\right]\right\}+\Gamma_{W Z}+\Gamma_{S B} .
$$

Here $f_{\pi}$ is the pion decay constant and $\epsilon$ is the dimensionless Skyrme parameter. $\Gamma_{W Z}$ is the Wess-Zumino action [20]:

$$
\Gamma_{W Z}=-\frac{i N_{C}}{240 \pi^{2}} \int_{M_{5}} d^{5} x \epsilon^{\mu \nu \rho \sigma \tau} \operatorname{Tr}\left[L_{\mu} L_{\nu} L_{\rho} L_{\sigma} L_{\tau}\right] \quad \text { with } \quad \partial M_{5}=M_{4} .
$$

Here we have used $L_{\mu}=U^{\dagger} \partial_{\mu} U$. The flavor symmetry breaking terms are contained in

$$
\begin{gathered}
\Gamma_{S B}=\int d^{4} x\left\{\frac{f_{\pi}^{2} m_{\pi}^{2}+2 f_{K}^{2} m_{K}^{2}}{12} \operatorname{Tr}\left[U+U^{\dagger}-2\right]+\frac{f_{\pi}^{2} m_{\pi}^{2}-f_{K}^{2} m_{K}^{2}}{2 \sqrt{3}} \operatorname{Tr}\left[\lambda_{8}\left(U+U^{\dagger}\right)\right]\right. \\
\left.+\frac{f_{K}^{2}-f_{\pi}^{2}}{4} \operatorname{Tr}\left[\hat{S}\left(U\left(\partial_{\mu} U\right)^{\dagger} \partial^{\mu} U+U^{\dagger} \partial_{\mu} U\left(\partial^{\mu} U\right)^{\dagger}\right)\right]\right\},
\end{gathered}
$$

where $\hat{S}=\operatorname{diag}(0,0,1)$ is the strangeness projector. It should be emphasized that many of the arguments presented below apply to more general chiral Lagrangians, though.

An appropriate parameterization of the collective coordinates in terms of eight "Eulerangles" is given by 


$$
A=D_{2}(\hat{I}) \mathrm{e}^{-i \nu \lambda_{4}} D_{2}(\hat{R}) \mathrm{e}^{-i(\rho / \sqrt{3}) \lambda_{8}},
$$

where $D_{2}$ denote rotation matrices of three Euler-angles for each, rotations in isospace $(\hat{I})$ and coordinate-space $(\hat{R})$. Substituting this configuration into the model Lagrangian yields upon canonical quantization the Hamiltonian for the collective coordinates $A$ :

$$
H=H_{\mathrm{s}}+\frac{3}{4} \gamma \sin ^{2} \nu
$$

The symmetric piece of this collective Hamiltonian only contains Casimir operators and may be expressed in terms of the $S U(3)$-right generators $R_{a}$, with $\left[A, R_{a}\right]=(1 / 2) A \lambda_{a}$, where $a=1, \ldots, 8$ :

$$
H_{\mathrm{s}}=M_{\mathrm{cl}}+\frac{1}{2 \alpha^{2}} \sum_{i=1}^{3} R_{i}^{2}+\frac{1}{2 \beta^{2}} \sum_{\alpha=4}^{7} R_{\alpha}^{2} .
$$

$M_{\mathrm{cl}}, \alpha^{2}, \beta^{2}$ and $\gamma$ are functionals of the soliton, $U_{0}(\vec{r})$. The field theoretical problem has been transformed into a quantum mechanical problem for the collective coordinates which are parameterized by the 'Euler-angles' (5). The symmetry breaking term in the Hamiltonian (6) depends on only one of the eight 'Euler-angles'. This suggests the following parameterization of the baryon eigenfunctions [15],

$$
\Psi_{I, I_{3}, Y ; J, J_{3}, Y_{R}}(A)=\frac{1}{\sqrt{N}} \sum_{M_{L}, M_{R}} D_{I_{3}, M_{L}}^{(I) *}(\hat{I}) f_{M_{L}, M_{R}}^{\left(I, Y ; J, Y_{R}\right)}(\nu) \mathrm{e}^{i Y_{R} \rho} D_{M_{R},-J_{3}}^{(J) *}(\hat{R}) .
$$

The unit baryon number sector constrains the right hypercharge to $Y_{R}=1$. This constraint stems form $\Gamma_{W Z}$ and is valid for $N_{C}=3$. The flavor hypercharge quantum number emerges via the constraint $Y-Y_{R}=2\left(M_{L}-M_{R}\right)$ for the intrinsic (iso-)spin projections $M_{L}$ and $M_{R}$.

The generators $R_{a}$ can be expressed in terms of derivatives with respect to the 'Eulerangles'. Then the eigenvalue problem $H \Psi=\epsilon \Psi$ reduces to sets of ordinary second order differential equations for the isoscalar functions $f_{M_{L}, M_{R}}^{\left(I, Y ; Y, Y_{R}\right)}(\nu)$. The product $\omega^{2}=\frac{3}{2} \gamma \beta^{2}$ appears as a continuous parameter in the eigenvalue equation. Hence the eigenfunctions (8) parametrically depend on $\omega^{2}$ which is thus interpreted as the effective strength of the flavor symmetry breaking. A value in the range $5 \lesssim \omega^{2} \lesssim 8$ is required to obtain reasonable agreement with the empirical mass differences for the $\frac{1}{2}^{+}$and $\frac{3}{2}^{+}$baryons [11]. In particular, reproducting the observed spacing $\left(M_{\Lambda}-M_{N}\right):\left(M_{\Sigma}-M_{\Lambda}\right):\left(M_{\Xi}-M_{\Sigma}\right)=1: 0.43$ : 0.69 demands a sizable $\omega^{2}$ since a leading order treatment of the eigenvalue equation (6) incorrectly yields $1: 1: \frac{1}{2}$. In the exact treatment we get significantly closer to the empirical values, e.g. for $\omega^{2}=6.0$ and $\omega^{2}=8.0$ we find the ratios $1: 0.69: 0.70$ and $1: 0.61: 0.77$, respectively!.

The symmetry breaking piece in eq (6) has non-zero matrix elements when sandwiched between baryon states that differ only by their respective $S U(3)$ representation. Hence the

\footnotetext{
${ }^{1}$ One might want to add other symmetry breaking operators to (6) but it should be reminded that they are of higher order in $1 / N_{C}$.
} 
exact as well as the perturbative treatments lead to baryon states which are not pure octet (or decuplet) states. Rather they have admxitures of states that are members of higher dimensional $S U(3)$ representations but otherwise have identical quantum numbers. Baryons in these representations cannot be constructed as three quark states, rather additional quarkantiquark pairs are required. Hence such admxitures to the octet (or decuplet) baryon wave-functions can be interpreted as an effective parameterization of the meson cloud. In general those admixtures reduce the baryon matrix elements associated with operators like $\bar{s} s$ [11]. Hence the meson cloud dominantly consists of pions. This merely reflects the fact that due to the smaller masses the non-strange degrees of freedom are easier to excite than those related to strange quarks. Here we are interested in the consequences that arise from the exact treatment of the collective coordinates. We therefore compare the admxitures of states from higher dimensional representations as they result from the exact calculation outlined above with those obtained in the first order approximation. As an example we list the admixture of the nucleon wave-functions with states carrying nucleon quantum numbers dwelling in higher dimensional $S U(3)$ representations in table $\mathbb{1}$. We observe that in the

TABLE II. The amplitude of various $S U(3)$ representations in the nucleon wave-functions. Presented are the exact and the first order results. In the exact treatment representations of higher dimensions than the $\overline{\mathbf{3 5}}$ also have non-vanishing amplitudes but they are not shown here.

\begin{tabular}{r|cccc|cccc}
\hline \hline & \multicolumn{9}{|c|}{ exact } & \multicolumn{4}{c}{ first order } \\
\hline$\omega^{2}$ & $\mathbf{8}$ & $\overline{\mathbf{1 0}}$ & $\mathbf{2 7}$ & $\overline{\mathbf{3 5}}$ & $\mathbf{8}$ & $\overline{\mathbf{1 0}}$ & $\mathbf{2 7}$ & $\mathbf{3 5}$ \\
\hline 4.0 & 0.977 & 0.170 & 0.128 & 0.018 & 1.000 & 0.200 & 0.130 & 0.000 \\
6.0 & 0.955 & 0.231 & 0.184 & 0.036 & 1.000 & 0.300 & 0.196 & 0.000 \\
8.0 & 0.927 & 0.278 & 0.233 & 0.056 & 1.000 & 0.400 & 0.261 & 0.000 \\
10.0 & 0.904 & 0.314 & 0.276 & 0.077 & 1.000 & 0.500 & 0.326 & 0.000 \\
\hline \hline
\end{tabular}

relevant range for $\omega^{2}$ the first order approximation has only limited validity. In particular the 10-amplitude is overestimated by this approximation.

The feature that the effective symmetry breaking parameter also contains the moment of inertia, $\beta^{2}$ for rotations into strangeness direction allows the possibility that the symmetry breaking in the wave-functions, which is measured by $\omega^{2}$, to be large albeit the explicit symmetry breaking, measured by $\gamma$, is not (and vice versa). Furthermore this allows for the scenario of having large deviations from flavor symmetric wave-functions without even having symmetry breaking components in the current operators since almost all symmetry breaking can eventually be included in non-derivative terms of $\Gamma_{S B}$ which do not contribute to currents. In the next section we will study whether such a picture can be consistent with the observations on hyperon beta-decays. These decays are well parameterized by the Cabibbo scheme [5] which is obtained by applying the Wigner-Eckart theorem to the $S U(3)$ symmetric baryon octet wave-functions.

\footnotetext{
${ }^{2}$ For example, the normalized nucleon matrix element $\langle N|\bar{s} s| N\rangle /\langle N|\bar{u} u+\bar{d} d+\bar{s} s| N\rangle$ reduces from $23 \%$ for the pure octet wave-function to $17 \%$ at $\omega^{2}=5.0$.
} 


\section{CHARGE OPERATORS}

In this section we present an investigation based on the covariance in the collective coordinate approach. In this context it is not necessary to detail the model Lagrangian. In the proceeding section we will nevertheless present an analysis which utilizes a specific Lagrangian as an example. It will be found that this example essentially verifies the results obtained from the covariant treatment.

Recently a chiral soliton model motivated analysis of the axial charges of the hyperons has been performed [21. Up to linear order in the strange current quark mass, $m_{s}$ (next-toleading order in flavor symmetry breaking) all operators for the respective matrix elements were collected. Their coefficients were determined from known data on hyperon beta-decay ${ }^{3}$. A model result was used to relate octet and singlet currents because they are not related by group theory. Then the polarization for the non-strange quarks in the $\Lambda$ was predicted to be small, $\Delta U_{\Lambda}=-0.02 \pm 0.17$ in contrast to $\Delta S_{\Lambda}=1.21 \pm 0.54$; with errors of the $\Xi$ decay data penetrating through this analysis. Some of the results (for the central values) raise questions in view of the study representing a perturbation expansion in flavor symmetry breaking: The axial singlet matrix element of the $\Lambda, \Delta \Sigma_{\Lambda}$, turned out to be about twice as large as that of the nucleon, $\Delta \Sigma_{N}$. Also, the $\mathcal{O}\left(m_{s}\right)$ terms contributed almost $50 \%$ to $\Delta S_{\Lambda}$. This indicates that at this order the expansion has not converged (if it does at all) or that in chiral soliton models the flavor symmetric point may not be the most suitable one to expand about. This may be perceived from the observation that in chiral soliton models the effect of the derivative type symmetry breaking terms is mainly indirect. They provide the splitting between the various decay constants and thus significantly increase $\gamma$ because it is proportional to $f_{K}^{2} m_{K}^{2}-f_{\pi}^{2} m_{\pi}^{2} \approx 1.5 f_{\pi}^{2}\left(m_{K}^{2}-m_{\pi}^{2}\right)$. Besides this indirect effect the derivative type symmetry breaking terms in (4) may be omitted. Whence there are no symmetry breaking terms in current operators and the octet axial charge operator may be parameterized as

$$
\int d^{3} r A_{i}^{(a)}=c_{1} D_{a i}-c_{2} D_{a 8} R_{i}+c_{3} \sum_{\alpha, \beta=4}^{7} d_{i \alpha \beta} D_{a \alpha} R_{\beta}, \quad a=1, \ldots, 8, \quad i=1,2,3 .
$$

Under flavor transformations (parameterized by changes of the collective coordinates) these charge operators behave like members of an octet. The expression (9) stems from subsituting the parameterization (1) into the covariant expression of the current operator obtained from a model Lagrangian using Noether's theorem and subsequently applying the quantization rules for the collective coordinates. Here we have furthermore introduced the adjoint representation of the collective rotations, $D_{a b}=\frac{1}{2} \operatorname{tr}\left(\lambda_{a} A \lambda_{b} A^{\dagger}\right)$. In principle, the constants $c_{n}, n=1,2,3$, are functionals of the soliton which can be computed within the adopted model. The $c_{2}$-term originates solely from the abnormal parity terms in the action, e.g. $\Gamma_{W Z}$, while the $c_{3}$-term additionally acquires contributions from field components which are

\footnotetext{
${ }^{3}$ Note that the standard definition for this decay parameter differs from that in ref [21] by a factor $\sqrt{6} / 2$.
} 
induced by the collective rotations. Both, $c_{2}$ and $c_{3}$ are subleading in $1 / N_{C}$ as the appearance of the generators, $R_{a}$ suggests. A well-known problem of many chiral soliton models is the too small prediction for the axial charge of the nucleon, $g_{A}$ when the constants $c_{n}$ are computed using the soliton solution. In this section we will not address that problem but rather use the empirical value $g_{A}=1.258$ as an input to determine the $c_{n}$. That is, we consider the constants $c_{n}$ as free parameters, alike $F \& D$ in the Cabibbo scheme.

It turns out that for pure octet wave-functions the matrix elements of the operators multiplying the constants $c_{1}$ and $c_{3}$ have the same ratio $F / D=5 / 9$ while the operator associated with $c_{2}$ has $F / D=-5 / 3$. This suggests to put $c_{1}+c_{3} / 2=-(3 F+5 D) / 2$ and $c_{2}=(9 F-5 D) / \sqrt{3}$ with the empirical values $g_{A}=F+D=1.258$ and $F / D=0.575 \pm 0.016$, i. e. $c_{1}+c_{3} / 2 \approx-2.69$ and $c_{2} \approx 0.09$. Of course, these relations are correct only for $\omega^{2}=0$. To see that the parameterization of the axial current matrix elements in terms of $F \& D$ Clebsch-Gordan coefficients becomes invalid already at moderate $\omega^{2}$ we consider the ratios $\left\langle B\left|D_{a 3}\right| B^{\prime}\right\rangle /\left\langle B\left|\sum_{\alpha, \beta=4}^{7} d_{3 \alpha \beta} D_{a \alpha} R_{\beta}\right| B^{\prime}\right\rangle$ in figure [1. The fact that the operators $D_{a 3}$ and

Ratio of Matrix Elements

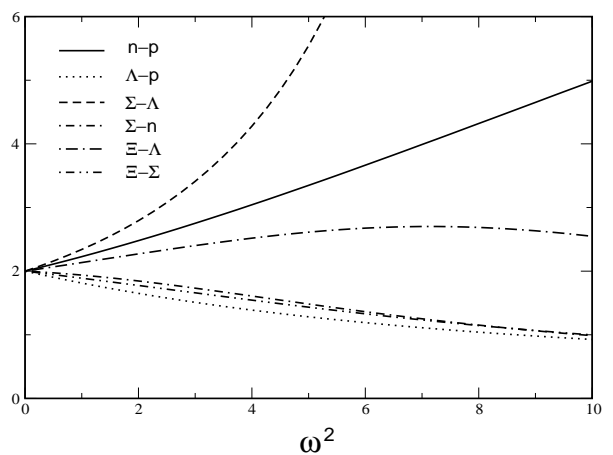

Vector Matrix Elements

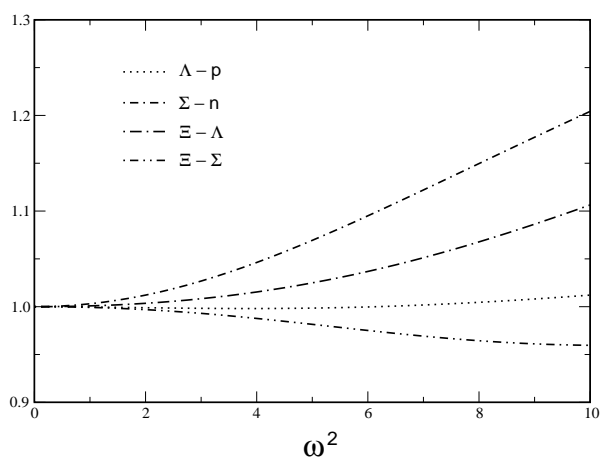

FIG. 1. The ratio of the matrix elements $\left\langle B\left|D_{a 3}\right| B^{\prime}\right\rangle /\left\langle B\left|\sum_{\alpha, \beta=4}^{7} d_{3 \alpha \beta} D_{a \alpha} R_{\beta}\right| B^{\prime}\right\rangle$ for the relevant baryon states $B$ and $B^{\prime}$ as a function of the effective symmetry breaking parameter $\omega^{2}$. The right panel shows the dependence of the vector matrix elements on symmetry breaking (12). They are normalized to the symmetric case.

$\sum_{\alpha, \beta=4}^{7} d_{3 \alpha \beta} D_{a \alpha} R_{\beta}$ have the same $F / D$ ratio is reflected by all ratios assuming the same value when flavor covariant wave-functions are used $\left(\omega^{2}=0\right)$. However, we see that already at moderate symmetry breaking the description of the axial current matrix elements in terms of $F / D$ ratios becomes inadequate as these operators evolve quite differently. With these significant dependencies on the effective symmetry breaking of matrix elements of the various operators contributing to the axial charges on $\omega^{2}$ it seems difficult to imagine that the empirical results for the hyperon decays, which are well described by the symmetric formulation, can be reasonably reproduced at realistic $\omega^{2} \gtrsim 5$.

Before attempting such a fit we can get more insight into the relevance of the constants $c_{n}$ from the axial singlet current. Although it is not related to the octet current (ब) by group theoretical means, the fact that within the collective coordinate approach we can consider flavor symmetry breaking as a continuous parameter provides further information. In the limit $\omega^{2} \rightarrow \infty$ (integrating out strange degrees of freedom) the model should reduce to the two flavor formulation. In particular the strangeness contribution to the axial charge of the nucleon should vanish in that limit. Noting that $\left\langle N\left|D_{83}\right| N\right\rangle \rightarrow 0$ and 
$\left\langle N\left|\sum_{\alpha, \beta=4}^{7} d_{3 \alpha \beta} D_{8 \alpha} R_{\beta}\right| N\right\rangle \rightarrow 0$ while $\left\langle N\left|D_{88}\right| N\right\rangle \rightarrow 1$ for $\omega^{2} \rightarrow \infty$, we demand

$$
\int d^{3} r A_{i}^{(0)}=-2 \sqrt{3} c_{2} R_{i} \quad i=1,2,3 .
$$

for the axial singlet current because it leads to the strangeness projection

$$
\begin{aligned}
\int d^{3} r A_{i}^{(s)} & =\frac{1}{3} \int d^{3} r\left(A_{i}^{(0)}-2 \sqrt{3} A_{i}^{(8)}\right) \\
& =\frac{-2}{\sqrt{3}}\left\{c_{1} D_{8 i}+c_{2}\left(1-D_{88}\right) R_{i}+c_{3} \sum_{\alpha, \beta=4}^{7} d_{i \alpha \beta} D_{8 \alpha} R_{\beta}\right\} .
\end{aligned}
$$

Actually all model calculations in the literature [22,23] are consistent with this requirement. It is simply a consequence of embedding the soliton in the isospin subgroup of flavor $S U(3)$. The analysis of the famous proton spin puzzle yielding $\Delta \Sigma_{N}=\left\langle N\left|\int d^{3} r A_{i}^{(0)}\right| N\right\rangle=0.20 \pm$ 0.10 then suggests $c_{2}=0.12 \pm 0.06$ in agreement with the above estimate from the flavor symmetric description of hyperon decays.

In order to completely describe the hyperon beta-decays we also demand matrix elements of the vector charges. These are obtained from the operator

$$
\int d^{3} r V_{0}^{(a)}=\sum_{b=1}^{8} D_{a b} R_{b}=L_{a}
$$

which introduces the left $S U(3)$ generators $L_{a}$. Again, this relation is obtained by substituting (11) into the covariant expression for the vector current operator extracted from the model Lagrangian. The relevant matrix elements are protected by the Ademollo-Gatto theorem [24] stating that deviations from the $S U(3)$ relations start at order $\left(\omega^{2}\right)^{2}$. In the collective coordinate appraoch this theorem is reproduced as the slope of these curves vanishes at $\omega^{2}=0, c f$. figure 1. Consequently, symmetry breaking in the vector currents is not only ignored in the Cabibbo scheme but also in the linear treatment of ref [21]. However, for the strongly distorted wave-functions, which we are utilizing, the deviations from the $S U(3)$ relations is sizable as is clearly shown in figure 1. Of course, we will take into account these deviations when computing the vector charges.

We now attempt to determine the constants $c_{n}$ to reasonably fit the ratios $g_{A} / g_{V}$ for the hyperon beta-decays (only $g_{A}$ for $\Sigma^{+} \rightarrow \Lambda e^{+} \nu_{e}$ ). The values for $g_{A}$ and $g_{V}$ are obtained from the appropriate matrix elements of respectively the operators in eqs (9) and (12), sandwiched between the eigenstates of the full Hamiltonian (6). We first have to fix a value, $\omega_{\text {fix }}^{2}$ for which we want to obtain the best fit. We adopt the following strategy: we choose $c_{2}$ according the proton spin puzzle and subsequently determine $c_{1}$ and $c_{3}$ at $\omega_{\text {fix }}^{2}=6.0$ such that the nucleon axial charge, $g_{A}$ and the $g_{A} / g_{V}$ ratio for $\Lambda \rightarrow p e^{-} \bar{\nu}_{e}$ are reproduced. For example, setting $\Delta \Sigma=0.2$ yields $c_{1}=-1.97, c_{2}=0.12$, and $c_{3}=-1.38$. This is not too different from the above consideration in the symmetric case as $c_{1}+c_{3} / 2=-2.66$. The matrix elements for the $n \rightarrow p$ and $\Lambda \rightarrow p$ transitions enter this determination of the $c_{n}$.

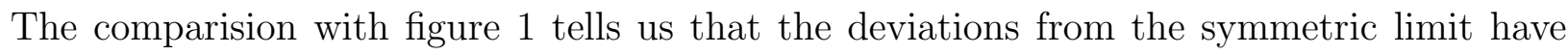
turned out unexpectedly small. We are now left with predictions not only for the decay parameters of the other decay processes but we can also study the variation with symmetry 

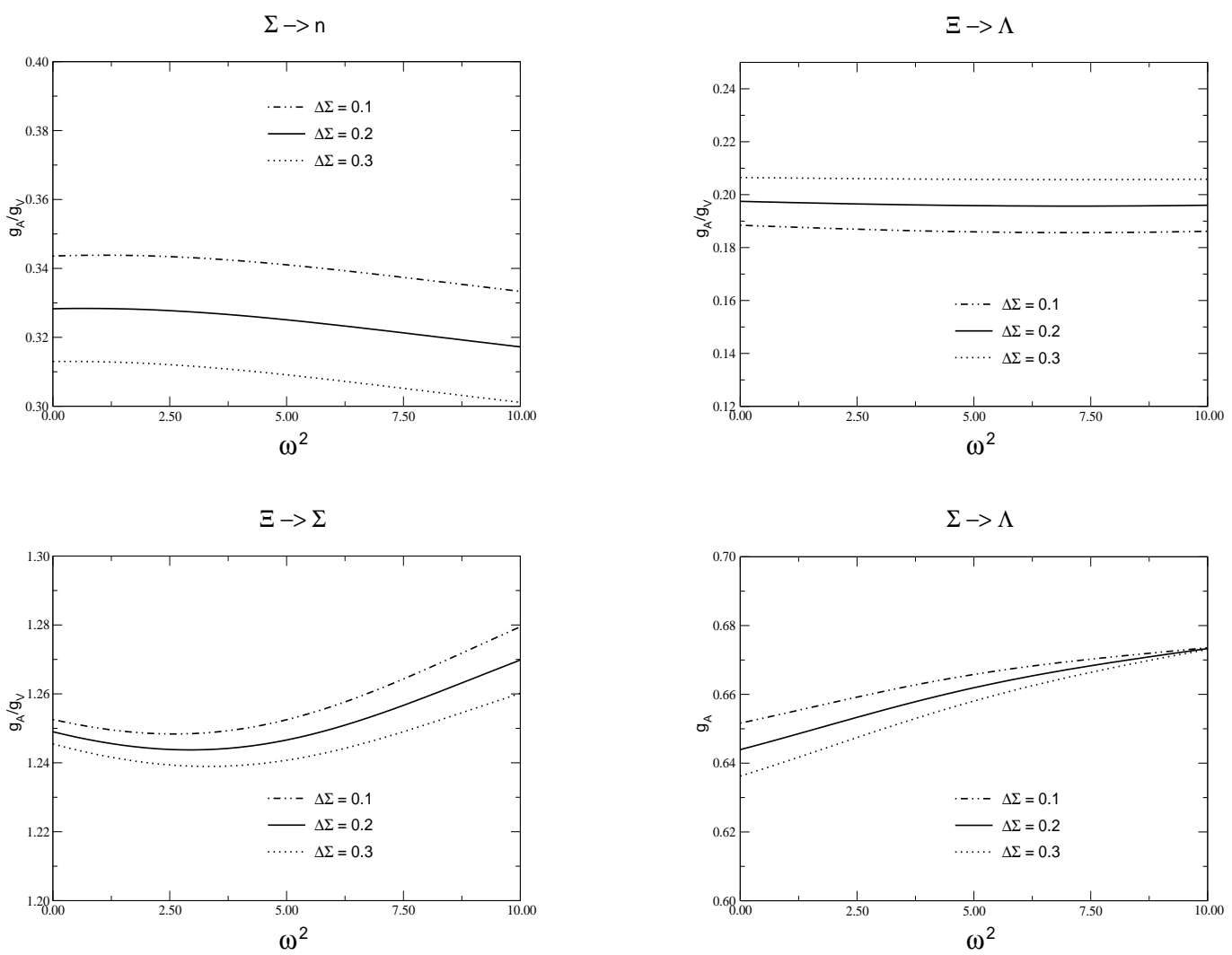

FIG. 2. The predicted decay parameters for the hyperon beta-decays using $\omega_{\text {fix }}^{2}=6.0$. The errors originating from those in $\Delta \Sigma_{N}$ are indicated.

breaking of all relevant decays. This is shown in figure 2. Obviously the dependence on flavor symmetry breaking is very moderate, on the order of only a few percent. In view of the model being an approximation this dependence may be considered irrelevant and the results

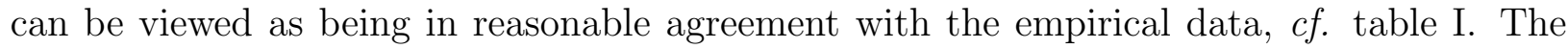
observed independence of $\omega^{2}$ shows that these predictions are not sensitive to the choice of $\omega_{\text {fix }}^{2}$. In addition, since we observe this approximate independence of $\omega^{2}$, we essentially have a two parameter $\left(c_{1}\right.$ and $c_{3}, c_{2}$ is fixed from $\left.\Delta \Sigma_{N}\right)$ fit of the hyperon beta-decays. This is alike the $F \& D$ parameterization in the Cabibbo scheme. We remark that the two transitions, $n \rightarrow p$ and $\Lambda \rightarrow p$, which are not shown in figure 2, exhibit a similar neglegible dependence on $\omega^{2}$ and, by construction, they match the empirical data at $\omega^{2}=6.0$. It should be noted that the use of the exact eigenfunctions of (6), which leads to the non-linear behavior is important in this regard. A linearized version (in $\omega^{2}$ ) would not have necessarily yielded this result. In particular a first order description would fail for the process $\Xi \rightarrow \Sigma$, for which $g_{A} / g_{V}$ is a non-monotonous function of $\omega^{2}$. Comparing the results shown in figure 2 with

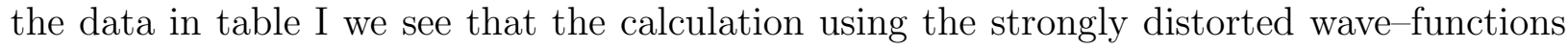
agrees approximately as well with the empirical data as the flavor symmetric $F \& D$ fit.

We also observe that the singlet current does not get modified. Hence we have the simple relation

$$
\Delta \Sigma_{N}=\Delta \Sigma_{\Lambda}
$$

for all values of $\omega^{2}$. 

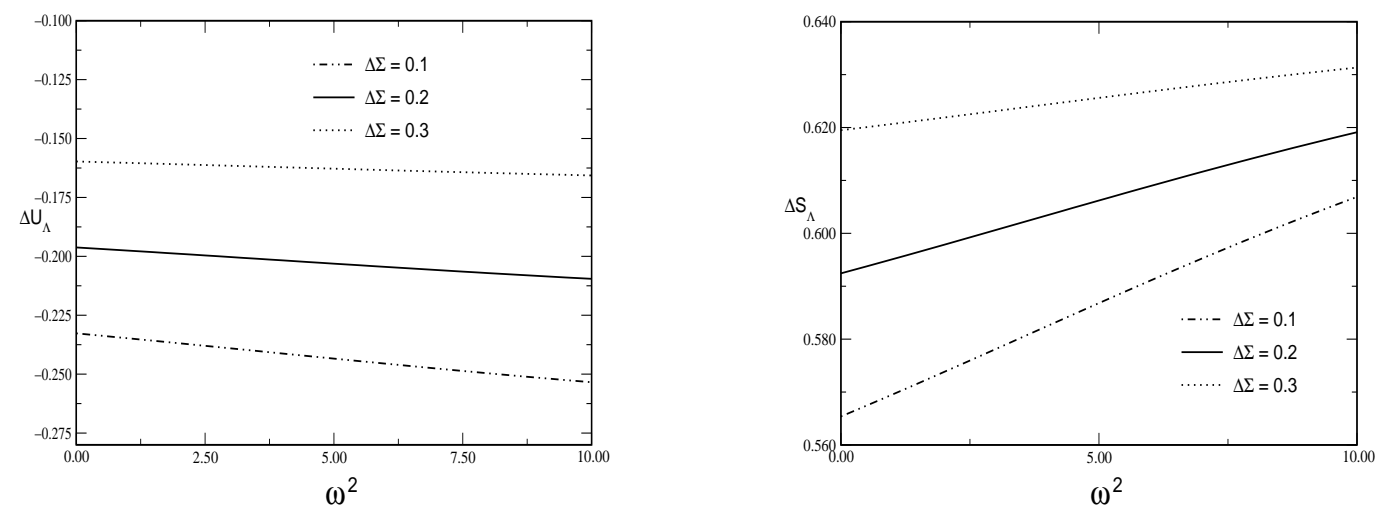

FIG. 3. The contributions of the non-strange (left panel) and strange (right panel) degrees of freedom to the axial charge of the $\Lambda$. Again we used $\omega_{\text {fix }}^{2}=6.0$.

In figure 3 we display the flavor components of the axial charge of the $\Lambda$ hyperon. We see that also the various contributions to the axial charge of the $\Lambda$ only exhibit a moderate dependence on $\omega^{2}$. The non-strange component, $\Delta U_{\Lambda}=\Delta D_{\Lambda}$ slightly increases in magnitude. The strange quark piece, $\Delta S_{\Lambda}$ then grows with symmetry breaking since we keep $\Delta \Sigma_{\Lambda}$ fixed. It should be remarked that the results shown in figure 3 agree nicely with an $S U(3)$ analysis applied to the data [1,2, 4]: $\Delta U_{\Lambda}=\Delta D_{\Lambda} \approx-0.20$ and $\Delta S_{\Lambda} \approx 0.60$. Finally we remark that the observed independence on the symmetry breaking does not occur for all matrix elements of the axial current. An interesting counter-example is the strange quark component in the nucleon, $\Delta S_{N}$. For $\Delta \Sigma=0.2$, say, it is significant at zero symmetry breaking, $\Delta S_{N}=-0.131$ while it decreases (in magnitude) to $\Delta S_{N}=-0.085$ at $\omega^{2}=6.0$.

Within this class of models the order of the expansion in symmetry breaking is measured by differences like $\omega^{2} \sim m_{K}^{2}-m_{\pi}^{2}, f_{K}^{2}-f_{\pi}^{2}$ or $m_{K^{*}}^{2}-m_{\rho}^{2}$ which are linear in the strange current quark mass $m_{s}$. In an systematic expansion one could add symmetry breaking components to the currents to eliminate the small deviations from the empirical data. As these corrections are potentially small it might well be that this could be accomplished by a single operator of $\mathcal{O}\left(m_{s}^{2}\right)$ or even higher. In turn this would make the approach quite

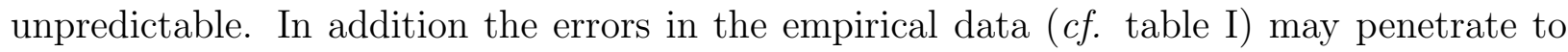
the fitted coefficients $c_{n}$. It seems thus more appropriate to revert to realistic models in which we can calculate the coefficients of the next-to-leading order terms and which have been tested at other instances.

\section{SPIN CONTENT OF THE $\Lambda$ IN A REALISTIC MODEL}

We consider a realistic soliton model which contains pseudoscalar and vector meson fields. It has been established for two flavors in ref [25] and been extended to three flavors in ref [22] where it has been shown to fairly describe the parameters of hyperon beta-decay (cf. table 4 in ref [22]).

Starting point is a three-flavor chirally invariant theory for pseudoscalar and vector mesons. The model Lagrangian contains terms which involve the Levi-Cevita tensor $\epsilon_{\mu \nu \rho \sigma}$, to accommodate processes like $\omega \rightarrow 3 \pi$ [26]. These terms contribute to $c_{2}$ and $c_{3}$. A minimal set of symmetry breaking terms is included [27] to account for different masses and decay 
constants. This effective theory contains topologically non-trivial static solutions, which are constructed by imposing ansätze in the isospin subgroup

$$
\xi(\vec{r})=\exp \left(\frac{i}{2} \hat{\vec{r}} \cdot \vec{\tau} F(r)\right), \quad \omega_{0}(\vec{r})=\omega(r) \quad \text { and } \quad \rho_{i, a}(\vec{r})=\frac{G(r)}{r} \epsilon_{i j a} \hat{r}_{j}
$$

while all other field components vanish classically. Here $\xi=\exp \left(i \vec{\pi} \cdot \vec{\tau} / 2 f_{\pi}\right)$ refers to the non-linear realization of the pion fields. The radial functions are determined by extremizing the static energy functional subject to boundary conditions appropriate to unit baryon number. Introducing collective coordinates for this configuration induces field components which are classically absent. From this, eight real radial functions emerge. They solve inhomogeneous linear differential equations with the soliton profiles (14) acting as sources. In regard of the discussion in the preceding section it is interesting to note that despite of strong symmetry breaking in the baryon wave-functions the model predictions for the magnetic moments approximately obey the respective $S U(3)$ relations [22].

Covariant expressions for the (axial-) vector currents are obtained by introducing appropriate sources. Substituting the above described ansätze and applying the quantization rules for the collective coordinates yields the charges as linear combinations of functionals, $c_{n}[F, \omega, G, \ldots]$ of the meson profile functions and operators in the space of the collective coordinates $A$. In this model the derivative type symmetry breaking terms add symmetry breaking pieces to the axial charge operator,

$$
\delta A_{i}^{(a)}=c_{4} D_{a 8} D_{8 i}+c_{5} \sum_{\alpha, \beta=4}^{7} d_{i \alpha \beta} D_{a \alpha} D_{8 \beta}+c_{6} D_{a i}\left(D_{88}-1\right) \quad \text { and } \quad \delta A_{i}^{(0)}=2 \sqrt{3} c_{4} D_{8 i} .
$$

The identical coefficient $c_{4}$ in the octet and singlet currents arises from the model calculations, it is not demanded by the above mentioned consistency condition of having vanishing strangeness contribution in the nucleon for $\omega^{2} \rightarrow \infty$ since we find $\left\langle N\left|D_{88} D_{83}\right| N\right\rangle \rightarrow 0$ as well as $\left\langle N\left|D_{83}\right| N\right\rangle \rightarrow 0$.

Once the model parameters are agreed on, the coefficients $c_{1}, \ldots, c_{6}$ are uniquely determined as are the parameters in the collective Hamiltonian, which in this model is more involved than eq (6). Thus the baryon wave-functions as well as the current operators are fixed and all relevant decay parameters can be computed. Unfortunately the model parameters cannot be completely determined in the meson sector [25]. We use the remaining freedom to accommodate baryon properties in three different ways as shown in table III]. The set denoted by 'b.f.' refers to an overall best fit to the spectrum of the low-lying spin $\frac{1}{2}$ and spin $\frac{3}{2}$ baryons. It predicts the axial charge somewhat on the low side, $g_{A}=0.88$. The set named 'mag.mom.' labels a set of parameters which yields magnetic moments which are close to the respective empirical data (with $g_{A}=0.98$ ) and finally the set labeled ' $g_{A}$ ' reproduces the axial charge of the nucleon and also reasonably accounts for hyperon betadecay [22]. For all three sets the effective symmetry breaking is sizable, $\omega^{2} \approx 10$. However, its effect is somewhat mitigated by additional symmetry breaking terms $\left(\sim \sum_{i=1}^{3} D_{8 i} R_{i}\right.$, $\left.\sum_{\alpha=4}^{7} D_{8 \alpha} R_{\alpha}\right)$ in the collective Hamiltonian (6). We observe that in particular the predictions for the axial properties of the $\Lambda$ are quite insensitive to the model parameters. The variation of the model parameters only seems to influence the isovector part of the axial charge operator. Surprisingly the singlet matrix element of the $\Lambda$ hyperon is smaller than 
TABLE III. Spin content of the $\Lambda$ in the realistic vector meson model. For comparison the nucleon results are also given. Three sets of model parameters are considered, see text.

\begin{tabular}{c||c|c|r||r|r|r|r}
\hline \hline \multicolumn{1}{c||}{} & \multicolumn{3}{c||}{$\Lambda$} & \multicolumn{4}{c}{$N$} \\
\hline & $\Delta U=\Delta D$ & $\Delta S$ & $\Delta \Sigma$ & $\Delta U$ & $\Delta D$ & $\Delta S$ & $\Delta \Sigma$ \\
\hline b.f. & -0.155 & 0.567 & 0.256 & 0.603 & -0.279 & -0.034 & 0.291 \\
mag. mom. & -0.166 & 0.570 & 0.238 & 0.636 & -0.341 & -0.030 & 0.265 \\
$g_{A}$ & -0.164 & 0.562 & 0.233 & 0.748 & -0.476 & -0.016 & 0.256 \\
\hline \hline
\end{tabular}

that of the nucleon, although this effect is tiny. As this difference emerges solely from the $c_{4}$ term this ordering is a reflection of $c_{4}$ being positive in this model. It should be noted that in other models $c_{4}$ is predicted to be negative [28], although small in magnitude as well; suggesting that $\Delta \Sigma_{\Lambda} \approx \Delta \Sigma_{N}$ in general.

Similar to the fit of the previous section the full model calculation predicts sizable polarizations of the up and down quarks in the $\Lambda$ which are slightly smaller in magnitude but nevertheless comparable to those obtained from the $S U(3)$ symmetric analysis. One wonders whether the significant up-quark polarization $\Delta U_{\Lambda}=\Delta D_{\Lambda} \approx-0.16$ has an experimental signature. The Gribov-Lipatov reciprocity relation [29] suggests that the quark fragmentation functions follow the quark distribution functions. Although there is no direct connection this hypothesis may nevertheless serve as an estimate [30,31]. As the integrated polarized distribution functions are just $\Delta Q_{B}$ (for quark $Q$ inside baryon $B$ ) it is suggestive that the predicted value for $\Delta U_{\Lambda}$ goes along with a significant up-quark fragmentation function for the $\Lambda$. In electroproduction the individual quark contributions to oberservables are weighted by the square of the respective charges. For the $\Lambda$ this elevates the non-strange contribution by a factor five. Equations (2)-(4) in ref [1] give the polarization of a $\Lambda$ that is produced in the current fragmentation region of deep inelastic electron-proton scattering. In addition to the (charge) ${ }^{2}$ factor the strange-quark contribution is suppressed in such processes as its distribution in the nucleon is presumably small. In essence the significant negative prediction for $\Delta U_{\Lambda}$ should result in a negative polarization of $\Lambda$ 's produced in that a reaction. In view of the self-analyzing decay $\Lambda \rightarrow p \pi^{-}$this should be detectable. However, it is subject to the (reasonable) assumption that fragmentation and distribution functions are closely related.

\section{CONCLUSIONS}

In the collective coordinate approach to chiral solitons large deviations from flavor symmetric (octet) wave-functions are required to accommodate the observed pattern of the baryon mass-splitting. Especially, contributions which arise beyond next-to-leading order in the effective symmetry breaking are needed for this purpose. In the QCD language these are of the order $m_{s}^{2}$ or higher. In this report we have suggested a picture for the axial charges of the low-lying $\frac{1}{2}^{+}$baryons which manages to reasonably reproduce the empirical data without introducing (significant) flavor symmetry breaking components in the corresponding operators. Rather, the sizable symmetry breaking resides almost completely in the baryon wave-functions. This scenario is especially motivated by the Yabu-Ando treatment 
of the Skyrme model which has the major symmetry breaking components in the potential part of the action and thus no (or only minor) symmetry breaking pieces in the current operators. The empirical data for these decay parameters are as reasonably reproduced as in the Cabibbo scheme of hyperon beta-decay. Repeatedly we emphasize that the present picture is not a re-application of the Cabibbo scheme since in the present calculation the 'octet' baryon wave-functions have significant admixture of higher dimensional representations ( $c f$. table II). Furthermore the individual matrix elements which enter this calculation may strongly vary with the effective symmetry breaking (or strange current quark mass), $c f$. figure 1; only when combining them to the full $g_{A} / g_{V}$ ratios the strong dependence on the strength of symmetry breaking cancels.

In the present treatment we may consider symmetry breaking as a continuous parameter. Taking this parameter to be infinitely large the two flavor model must be retrieved for the nucleon. This consistency condition relates coefficients in the axial singlet current operator to the respective octet components, which are not otherwise related to each other by group theory. In turn we are enabled to completely disentangle the quark flavor components of the axial charge. It results in sizable up and down quark polarizations in the $\Lambda$. Again, a picture emerged which, after some cancellations, agrees with that of the flavor symmetric treatment for known data. These results were obtained utilizing a parameterization of a charge operator which did not contain any symmetry breaking component.

We have also considered a realistic model, wherein the parameters entering the charge operators are actually predicted. These operators contain non-vanishing symmetry breaking pieces, whose matrix elements are, however, small. Essentially this model calculation confirmed the results obtained in the parametrically treatment.

\section{Acknowledgments}

The author would like to thank G. R. Goldstein, R. L. Jaffe and J. Schechter for helpful conversations and useful references.

This work is supported in part by funds provided by the U.S. Department of Energy (D.O.E.) under cooperative research agreement \#DF-FC02-94ER40818 and the Deutsche Forschungsgemeinschaft (DFG) under contract We 1254/3-1. 


\section{REFERENCES}

[1] R. L. Jaffe, Phys. Rev. D54 (1996) 6581.

[2] M. Burkardt and R. L. Jaffe, Phys. Rev. Lett. 70 (1993) 2537.

[3] D. de Florian, M. Stratmann, and W. Vogelsang, hep-ph/9710410: Polarized Lambda Production at HERA.

[4] C. Boros and L. Zuo-tang, Phys. Rev. D57 (1998) 4491; and references therein.

[5] N. Cabibbo, Phys. Rev. Lett. 10 (1963) 531.

[6] R. Flores-Mendieta, E. Jenkins, and A. V. Manohar, Phys. Rev. D58 (1998) 094028.

[7] C. Caso et al., (Particle Data Group), Eur. Phys. J. C3 (1998) 1, M. Bourquin et al., Z. Phys. C12 (1982) 307; C21 (1983) 1.

[8] N. W. Park, J. Schechter, and H. Weigel, Phys. Rev. D41 (1990) 2836.

[9] S. Brodsky, J. Ellis, and M. Karliner, Phys. Lett. B206 (1988) 309.

[10] R. Johnson, N. W. Park, J. Schechter, V. Soni, and H. Weigel, Phys. Rev. D42 (1990) 2998.

[11] H. Weigel, Int. J. Mod. Phys. A11 (1996) 2419; J. Schechter and H. Weigel, hep ph/9907554: The Skyrme Model for Baryons.

[12] M. Praszałowicz, Phys. Lett. 158B (1983) 264.

[13] M. Chemtob, Nucl. Phys. B256 (1985) 600.

[14] J. Donoghue and C. R. Nappi, Phys. Lett. 168B (1986) 105.

[15] H. Yabu and K. Ando, Nucl. Phys. B301, 601 (1988).

[16] N. W. Park, J. Schechter, and H. Weigel, Phys. Lett. B228 (1989) 420.

[17] W. Lu and B. Q. Ma, Phys. Lett. B357 (1995) 419.

[18] J. Ellis, D. Kharzeev, and A. Kotzinan, Z. Phys. C69 (1996) 467.

[19] M. Karliner and H. J. Lipkin, Phys. Lett. B461 (1999) 280.

[20] E. Witten, Nucl. Phys. B223 (1983) 422; Ö. Kaymakcalan, S. Rajeev and J. Schechter, Phys. Rev. D30 (1984) 594.

[21] H.-C. Kim, M. Praszałowicz, and K. Goeke, Acta Phys. Polon. B31 (2000) 1767; see also H.-C. Kim, M. Praszałowicz, and K. Goeke, Phys. Rev. D61 (2000) 114006.

[22] N. W. Park and H. Weigel, Nucl. Phys. A541 (1992) 453.

[23] A. Blotz, M. Praszałowicz, and K. Goeke, Phys. Lett. B317 (1993) 195.

[24] M. Ademollo and R. Gatto, Phys. Rev. Lett. 13 (1964) 264.

[25] P. Jain, R. Johnson, Ulf-G. Meißner, N. W. Park, and J. Schechter, Phys. Rev. D37 (1988) 3252; Ulf-G. Meißner, N. Kaiser, H. Weigel, and J. Schechter, Phys. Rev. D39 (1989) 1956.

[26] Ö. Kaymakcalan, S. Rajeev, and J. Schechter, Phys. Rev. D30 (1984) 594.

[27] P. Jain, R. Johnson, N. W. Park, J. Schechter, and H. Weigel, Phys. Rev. D 40 (1989) 855.

[28] H. Weigel, R. Alkofer, and H. Reinhardt, Nucl. Phys. B387 (1992) 638; A. Blotz, D. Diakonov, K. Goeke, N. W. Park, V. Petrov, and P. V. Pobylitsa, Nucl. Phys. A555 (1993) 765.

[29] V. N. Gribov and L. N. Lipatov, Phys. Lett. B37 (1971) 78.

[30] S. J. Brodsky and B.-Q. Ma, Phys. Lett. 392 (1997) 542.

[31] C. Boros and A. W. Thomas, Phys. Rev. D60 (1999) 074071. 